\section{Condição periodontal de um subgrupo populacional do município de Guarulhos, SP}

\section{Periodontal status of a population subgroup from Guarulhos, SP}

Fátima de Lourdes Bolzan Pion'

Marcelo Werneck Barata de Araujo ${ }^{2}$

Magda Feres ${ }^{3}$

\section{Sheila Cavalca Cortelli ${ }^{4}$}

${ }^{1}$ Mestre em Odontologia, Área de Concentração em Periodontia, Universidade Guarulhos

${ }^{2}$ Oral Care R\&D, Pfizer Consumer Healthcare

${ }^{3}$ Professora Titular de Periodontia da Universidade Guarulhos

${ }^{4}$ Professora Adjunta de Periodontia da Universidade Guarulhos

Correspondência: Fátima de Lourdes Bolzan Pion. Rua Monte Alegre, 523 conjunto 71 Perdizes. São Paulo, SP CEP 05014-000,E-mail: fatimapc@uol.com.br

\section{Resumo}

Este estudo transversal determinou a condição periodontal e sua associação com fatores de risco em uma amostra de conveniência de 588 indivíduos atendidos na Universidade Guarulhos, entre 1999 e 2003. Características geográficas, demográficas e comportamentais, parâmetros bucais e periodontais foram tabulados e analisados em SPSS 11.5. Os testes $t$ Student e Qui-quadrado determinaram a significância $(p<0,05)$ das variáveis contínuas e categóricas. O teste de correlação de Pearson correlacionou a ocorrência de doença (PS média $>3,0 \mathrm{~mm}$ ) com fatores de risco (por exemplo: idade, sexo, fumo) e comportamentais. A análise de regressão linear multivariada determinou a associação entre exposição aos fatores de risco e doença. A população estudada com média de idade de 36,1 $\pm 14,5$ anos foi composta principalmente por mulheres $(72,1 \%)$ e minoria fumante $(13,3 \%)$. Os valores médios de dentes ausentes e profundidade de sondagem (PS) foram de 5,7 $\pm 5,9$ dentes e $2,2 \pm 0,8 \mathrm{~mm}$, respectivamente. A prevalência de PS > 3,0mm (valor referência) foi de $13,5 \%$; e a distribuição regional da população de acordo com este critério foi: centro, 2,0\%; zona oeste, 17,8\%; zona leste, $38,0 \%$; zona sul, $6,7 \%$; e zona norte, $35,5 \%$. As freqüências médias dos índices de placa e sangramento a sondagem foram de $75,3 \%$ e $34,1 \%$, respectivamente. A condição periodontal exibiu correlação com idade, fumo e presença de biofilme supragengival. Exceto pela última, estas variáveis também mostraram associação pela análise de regressão multivariada. A população estudada exibiu elevado grau de inflamação gengival e baixo percentual de bolsas periodontais, estando a condição periodontal associada à idade e ao hábito de fumar.

Palavras-chave: Estudos epidemiológicos. Doenças periodontais. Ficha clínica. Fatores de risco. 
Abstract

This cross-sectional study determined the periodontal status and its association with risk factors in a convenience sample of 588 subjects seen at the dental clinic of Guarulhos University from 1999 to 2003. To reach the study objectives, geographic, demographic and behavioral characteristics, as well as, oral and periodontal parameters were tabulated and analyzed using SPSS. Chi-square and Student's $t$ tests determined significance $(\mathrm{p}<0.05)$ of continuous and categorical variables. Pearson's correlation test correlated disease (PPD mean $>3 \mathrm{~mm}$ ) with risk (age, sex, smoking) and behavioral factors. Multivariate linear regression evaluated the association between risk factors and periodontal disease. The study population presented a mean age of $36.1 \pm 14.5$ years, and was primarily comprised women $(72.1 \%)$, while smokers were minority $(13.3 \%)$. The mean number of missing teeth and periodontal probing depth (PPD) were $5.7 \pm 5.9$ teeth and $2.2 \pm 0.8 \mathrm{~mm}$, respectively. The prevalence of PPD $>3.0 \mathrm{~mm}$ was $13.5 \%$ and the regional distribution of the population following the same cut-off point was: center $2.0 \%$, west zone $17.8 \%$, east zone $38.0 \%$, south zone $6.7 \%$, and north zone $35.5 \%$. The average indices for plaque and bleeding on probing were $75.3 \%$ and $34.1 \%$, respectively. Periodontal status was correlated with age, smoking habits and presence of supragingival biofilm. However, the latter did not show association when entered on the multivariate analysis, in contrast with the others. The study population showed high levels of gingival inflammation and a low percentage of periodontal pockets. Periodontal status was associated with smoking habits and age.

Keywords: Epidemiological studies. Periodontal diseases. Clinical record. Risk factors.

\section{Introdução}

O termo doença periodontal refere-se a diferentes quadros clínicos denominados doenças gengivais ou gengivite quando limitados aos tecidos de proteção, e denominados periodontite quando acometem os tecidos de suporte do elemento dentário. Assim, a periodontite caracteriza-se por perda de inserção progressiva, incluindo destruição do ligamento periodontal e suporte ósseo alveolar com conseqüente formação de bolsa periodontal, o que ocorre devido ao acúmulo microbiano modulado por uma série de fatores ambientais, locais e sistêmicos ${ }^{1}$.

Guarulhos é um município da grande São Paulo e representa a $8^{\text {a }}$ economia do Estado. Com 1.218.862 habitantes e uma área territorial de $318 \mathrm{Km}^{2}$, dispõe de 291 equipos odontológicos públicos (1 equipo/ 4.188,5 habitantes). Em 2000, época de realização do último censo pelo Instituto Brasileiro de Geografia e Estatística - IBGE ${ }^{2}$, contava com 1.072 .717 habitantes, $49,2 \%$ do sexo masculino e $50,8 \%$ do sexo feminino. Em relação à etnia, nesta época, $62,1 \%$ dos entrevistados se autoclassificaram como brancos, $30,9 \%$ como pardos e $5 \%$ como pretos, enquanto amarelos e índios totalizaram 1,3\% dos entrevistados.

Os estudos transversais ou seccionais caracterizam-se por uma observação direta de uma determinada população em uma única oportunidade. Os objetivos desse tipo de estudo estão sempre relacionados com indivíduos em determinados locais e em determinadas épocas. Todavia, devese mencionar que esta relação temporal entre causa e efeito é dependente da memória do indivíduo. Por isso, nem sempre se consegue estabelecer com sucesso a relação entre doença e exposição aos fatores de risco. Assim, esse tipo de estudo possibilita a inferência dos resultados observados para uma população definida no tempo e no espaço, sendo por isso amplamente utilizado para investigar doenças crônicas, de início indeterminado e de longa duração, ou ainda para doenças que 
sofrem variações de intensidade, ao longo da vida, como as de início precoce ${ }^{3,4}$.

Devido à dificuldade de observação direta e mensuração das exposições em grande número de indivíduos, os estudos ecológicos podem representar uma alternativa com custo bastante reduzido, desde que se utilizem dados agrupados de forma homogênea ${ }^{3}$. Devido à possibilidade de coletar informações por meio de diferentes metodologias cria-se uma expectativa favorável em relação a uma ampla disponibilidade de dados epidemiológicos. Entretanto, para a doença periodontal, a literatura nacional ainda é escassa, principalmente ao considerarmos a extensão territorial do Brasil. Em 2002, o periódico Periodontology 2000 designou um número inteiro à abordagem de dados epidemiológicos mundiais e, no artigo sobre as Américas Central e do Sul, os dados brasileiros perfizeram $23,33 \%$ das publicações referenciadas, as quais foram levantadas em bases de dados brasileiras e internacionais ${ }^{5}$. Além disso, a carência de dados também pode ser evidenciada se mencionarmos que poucos levantamentos epidemiológicos de base nacional, como o de $1986^{6}$ e o projeto SB - Brasil $2003^{7}$, têm sido realizados de modo a incluir na análise a condição periodontal.

Outro problema, não apenas relacionado à epidemiologia periodontal no Brasil, é a grande divergência no que se refere à definição de doença (Quadro 1), considerando-se que tais critérios exercem influência direta sobre os resultados observados. Além disso, deve-se adicionar à diversidade de critérios a influência de determinantes e fatores de risco tanto na manifestação como na progressão da doença periodontal. Neste contexto, estudos transversais e longitudinais têm demonstrado associação entre ocorrência e gravidade da doença periodontal com o aumento da idade ${ }^{8-10}$, o sexo masculino ${ }^{8-10}$, a afrodescendência ${ }^{11} \mathrm{e}$ a condição socioeconômica. Esta última parece influenciar de modo mais direto a gengivite $^{12,13}$ do que a periodontite ${ }^{14-16}$. Além destes fatores, fortes evidências também têm sugerido associação entre diabetes mellitus e consumo de cigarros com a condição periodontal ${ }^{10,17}$.

O objetivo geral do presente estudo foi determinar a condição periodontal de um subgrupo populacional de Guarulhos. E os objetivos específicos foram: 1) indicar as características demográficas e geográficas da população estudada; 2) determinar quais os fatores de risco que influenciaram a condição periodontal nesta população.

\section{Materiais e métodos}

\section{Coleta de dados}

O protocolo de pesquisa do presente estudo foi avaliado e aprovado pelo Comitê de Ética em Pesquisa envolvendo seres humanos da Instituição (Universidade Guarulhos), anteriormente à coleta de dados (MR01/03). O número do prontuário como identificador foi adotado tanto para a aleatorização da amostra, executada com auxílio de uma tabela de números equiprováveis, como para manter sob sigilo a identidade dos participantes. A partir dos 24.000 prontuários disponíveis na Instituição, estabelecendo-se uma potência de $99 \%$ e p < 0,01, determinou-se como satisfatória uma amostra aleatória composta por 500 indivíduos. Para atingir o número proposto foram analisados 968 prontuários, dos quais 380 foram excluídos, ou por não estarem completos e adequadamente preenchidos $(96,05 \%)$, ou por se referirem a indivíduos não residentes em Guarulhos (3,94\%). Assim, nossa amostra de conveniência foi composta por 588 moradores de Guarulhos atendidos no Curso de Odontologia da Universidade Guarulhos, no período de 1999 a 2003, e o banco de dados gerado incluiu 134.064 informações (228 variáveis) coletadas pelos alunos de graduação deste período. As variáveis periodontais avaliadas com auxílio de sonda periodontal manual modelo Carolina do Norte foram: Profundidade de Sondagem - PS (mm: 6 sítios/dente), Índice de placa - IP $(0,1: 4$ 


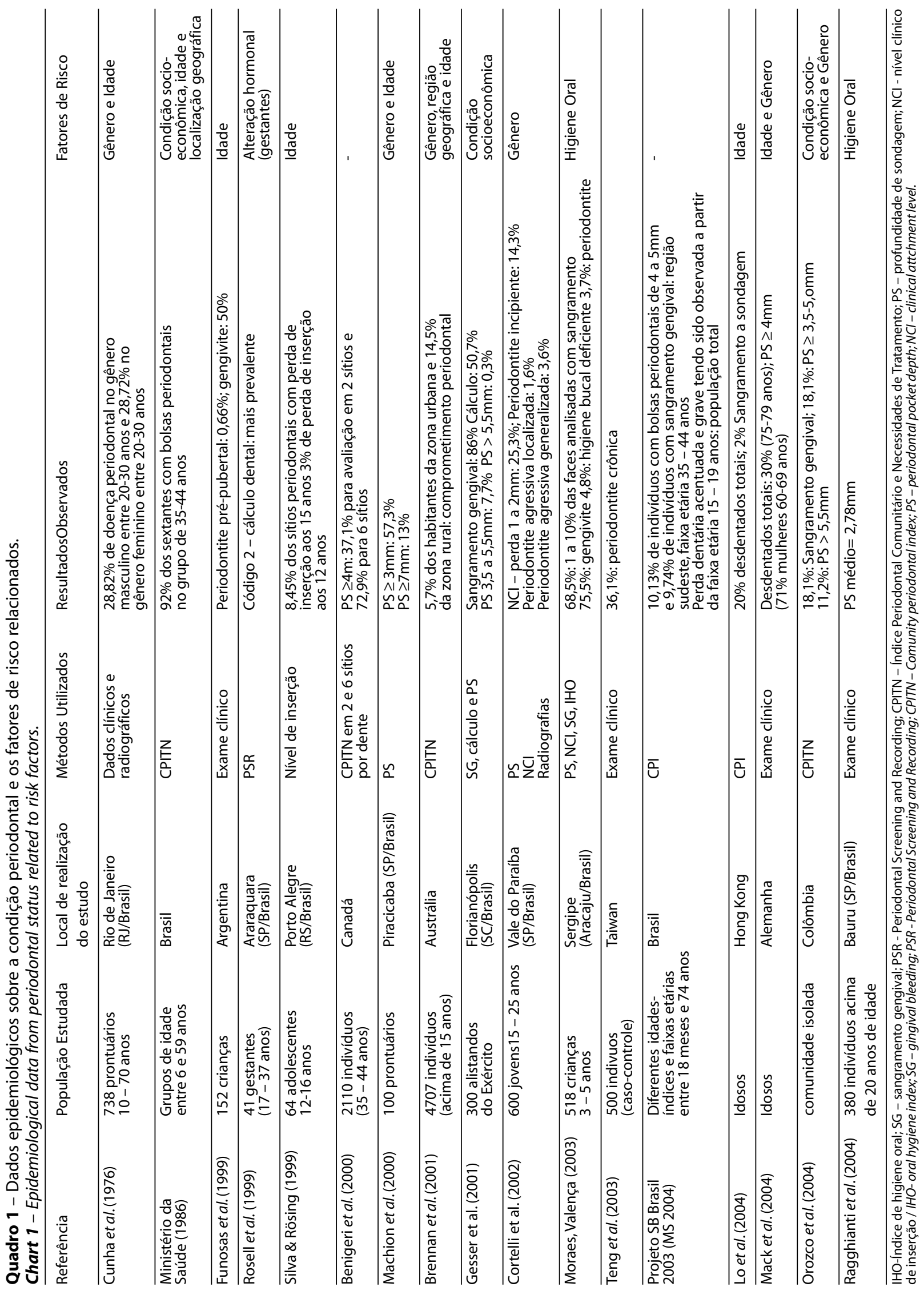

\begin{tabular}{c|l|l}
\hline Rev Bras Epidemiol & 338 & Condição periodontal de um subgrupo populacional do município de Guarulhos, SP \\
\hline 2006; 9(3): 335-45 &
\end{tabular} 
sítios/dente) e Sangramento à Sondagem - SS (0,1: 4 sítios/dente). As características demográficas, geográficas e comportamentais foram: idade e sexo (ambos de acordo com o documento de identificação apresentado), etnia (autoclassificação), hábitos (relativos ao consumo de tabaco, controle mecânico e químico do biofilme supragengival), história médicoodontológica incluindo doenças sistêmicas (crônicas como diabetes mellitus, cardiopatias e desordens renais; infecciosas como síndrome da imunodeficiência humana adquirida, sífilis; além de doenças típicas da infância), saúde da mulher (incluindo dados sobre o número de gestações e partos, menarca, menopausa e osteoporose), tratamentos prévios sistêmicos ou odontológicos de qualquer natureza (cirúrgicos ou não, periodontal ou dentário, incluindo a utilização de antibióticos, antiinflamatórios e imunossupressores), e exames gerais de avaliação da condição de saúde (como glicemia de jejum e/ou pós-prandial, eletroencefalograma e ultrassonografia). De acordo com o endereço residencial fornecido pelo participante foi levantado o CEP do logradouro, que foi então relacionado a um bairro específico. Finalmente, foi verificada a localização deste bairro de acordo com o zoneamento municipal de Guarulhos, SP.

\section{Análise estatística}

A variável PS originou um valor médio/ indivíduo e, foi definido como doença periodontal a média bucal de PS > 3,0 $\mathrm{mm}$ (AAP, 1996 - critério modificado). Os índices IP e SS foram considerados da seguinte forma: presença ou ausência de biofilme ou sangramento a sondagem/dente e percentual de dentes com biofilme ou sangramento a sondagem/indivíduo. Determinou-se, por meio do teste $t$ - Student e Qui-quadrado, a significância estatística das variáveis contínuas (PS) e categóricas (IP, SS) $(\mathrm{p}<0,05)$

O teste de correlação de Pearson foi utilizado para correlacionar a ocorrência de doença (de acordo com PS) com fatores de risco (idade, sexo, hábito de fumar, diabete) e comportamentais (hábitos de higiene bucal). As variáveis com significância estatística ( $t$ Student e Qui-quadrado), foram submetidas a análise de regressão linear multivariada, para determinar a associação entre exposição aos fatores de risco e doença. Desta forma, as variáveis que não apresentaram associação estatística significativa com doença periodontal não foram incluídas no modelo de regressão. Todos os dados foram analisados com auxílio do software SPSS ${ }^{\circledR} 11.5$ e o cálculo amostral determinado pelo programa PS - Power and Sample Size 2.1.30.

\section{Resultados}

\section{Condição periodontal, características demográficas, geográficas e comporta- mentais}

Foram observados indivíduos entre 10 e 76 anos de idade $(36,1 \pm 14,5)$, sendo $71,3 \%$ brancos, $5,8 \%$ pretos, $13,6 \%$ pardos, e $9,3 \%$ relataram que desconheciam sua classificação em relação à etnia. A população foi composta por $72,1 \%$ de indivíduos do sexo feminino e $27,9 \%$ do sexo masculino. A população estudada residia na sua maioria (97\%) na zona urbana e principalmente na zona leste $(37,7 \%)$. Observou-se uma ampla variabilidade em relação ao tempo de exposição ao hábito de fumar (de 1 a 40 anos com média populacional de 2,5 $\pm 6,8$ anos), assim como em relação à quantidade de cigarros consumida (de 2 a 40 cigarros com média populacional de 2,81 \pm 10 cigarros/dia), principalmente ao se considerar o baixo percentual de fumantes $(13,3 \%)$ encontrados.

A população apresentou média de 2,72 $\pm 0,85$ escovações por dia, $48,3 \%$ usavam fio dental e poucos indivíduos $(23,7 \%)$ já haviam sido submetidos a algum tipo de terapia periodontal. A grande maioria dos indivíduos $(86,5 \%)$ apresentou valores 
médios de PS $\leq 3 \mathrm{~mm}$, e somente 13,5\% PS $>3 \mathrm{~mm}$. Foi observado acúmulo elevado de biofilme e ausência de diferenças significativas entre sexos para os parâmetros PS, IP, SS (Tabela 1). Os dados de anamnese relacionados ao valor referência de profundidade de sondagem (PS > 3mm) estão descritos na Tabela 2.

Quando da estratificação por zonas urbanas, 38,0\% dos indivíduos provenientes da zona leste, $35,5 \%$ da zona norte, $17,8 \%$ da zona oeste, $6,7 \%$ da zona sul e $2,0 \%$ da zona central, apresentaram doença periodontal. Das zonas de exclusão socioeconômica, leste e oeste, apenas a primeira exibiu maior prevalência ( $\mathrm{p}<$ $0,05)$ de doença periodontal em relação às demais áreas geográficas.

\section{Fatores de risco e condição periodontal}

O fator idade exibiu correlação com ausência dentária $(\mathrm{r}=0,501$ e $\mathrm{p}<0,01)$ e quantidade de cigarros consumidos durante a vida $(r=0,133$ e $\mathrm{p}<0,01)$, enquanto o acúmulo de biofilme supragengival (IP) foi acompanhado por maior ocorrência de SS (Tabela 3). A PS exibiu correlação com idade, tempo de exposição ao hábito de fumar, ausência dentária, IP e com o número de dentes presentes na cavidade bucal (Tabela 3). A Figura 1 traz a representação gráfica da dispersão entre a variável de interesse (PS) com idade e ausência dentária. As correlações significativas encontradas entre PS e informações coletadas durante a anamnese estão expressas na Tabela 4.

A análise de Regressão Linear multivariada mostrou associação entre PS $>3 \mathrm{~mm}$ com idade, hábito de fumar, SS e realização prévia de teste glicêmico (Tabela 5).

\section{Discussão}

Aspectos demográficos da população

Tabela 1 - Dados das 588 fichas clínicas relativas à condição periodontal. Guarulhos, SP, 19992003.

Table 1 - Data from 588 clinical records related to periodontal status. Guarulhos, SP, 1999-2003.

\begin{tabular}{lccc}
\hline Variável & \multicolumn{3}{c}{ Média \pm desvio padrão } \\
& Sexo feminino & Sexo masculino & Total \\
\hline Profundidade de sondagem (mm) & $2,2 \pm 0,8$ & $2,1 \pm 0,7$ & $2,2 \pm 0,8$ \\
Índice de placa (\%) & $74,3 \pm 23,1$ & $75,4 \pm 23,7$ & $75,3 \pm 23,6$ \\
Sangramento à sondagem (\%) & $33,7 \pm 25,5$ & $36,4 \pm 27,9$ & $34,1 \pm 26,1$ \\
\hline
\end{tabular}

Tabela 2 - Dados da anamnese relacionados ao valor referência de profundidade de sondagem. Guarulhos, SP, 1999-2003.

Table 2 - Clinical information related to probing depth cut-off. Guarulhos, SP, 1999-2003.

\begin{tabular}{lcccc}
\hline Condição periodontal & \multicolumn{2}{c}{ PS $>3 \mathrm{~mm}(\mathrm{n}=82)$} & \multicolumn{2}{c}{ PS $£$ 3mm(n=506) } \\
& Sim (\%) & Não (\%) & Sim (\%) & Não (\%) \\
\hline Visita ao Cirurgião-dentista & 6,2 & 93,8 & 12,8 & 87,2 \\
Uso de anti-séptico & 4,0 & 96,0 & 11,6 & 88,4 \\
Sangramento gengival & 9,0 & 91,0 & 13,3 & 86,7 \\
Irritação gengival & 7,0 & 93,0 & 13,2 & 86,8 \\
Tratamento periodontal prévio & 4,5 & 95,5 & 13,5 & 86,5 \\
Hábito de fumar & 2,4 & 97,6 & 13,4 & 86,6 \\
Uso de fio dental & 13,3 & 86,7 & 11,1 & 88,9 \\
\hline
\end{tabular}

PS - profundidade de sondagem (média/indivíduo); $\mathrm{n}$ = número de indivíduos.

$P S$ - periodontal probing depth (mean/subject); $n=$ number of subjects 
Tabela 3 - Correlação entre condição periodontal e fatores de risco associados.

Table 3 - Correlation between periodontal status and risk factors.

\begin{tabular}{|c|c|c|c|c|c|c|c|c|c|}
\hline & $\begin{array}{c}\text { Última consulta } \\
\text { odontológica } \\
\text { (meses) }\end{array}$ & $\begin{array}{c}\text { Escovação } \\
\text { dental/dia } \\
\text { (n) }\end{array}$ & $\begin{array}{l}\text { Cigarros/ } \\
\text { dia } \\
\text { (n) }\end{array}$ & $\begin{array}{l}\text { Consumo } \\
\text { de cigarros } \\
\text { (anos) }\end{array}$ & $\begin{array}{l}\text { Cigarros/ } \\
\text { vida } \\
\text { (n) }\end{array}$ & $\begin{array}{c}\text { Dentes } \\
\text { presentes } \\
\text { (n) }\end{array}$ & $\begin{array}{c}\mathrm{IP} \\
\text { (freqüência) }\end{array}$ & $\begin{array}{c}\text { SS } \\
\text { (freqüência) }\end{array}$ & $\begin{array}{c}\text { PS } \\
\text { (média) }\end{array}$ \\
\hline $\begin{array}{l}\text { Última consulta } \\
\text { odontológica } \\
\text { (meses) }\end{array}$ & 1 & & & & & & & & \\
\hline $\begin{array}{l}\text { Escovação dental/ } \\
\text { dia (n) }\end{array}$ & 0,024 & 1 & & & & & & & \\
\hline Cigarros/ dia (n) & $-0,017$ & $-0,053$ & 1 & & & & & & \\
\hline $\begin{array}{l}\text { Consumo de } \\
\text { cigarros (anos) }\end{array}$ & $-0,022$ & $-0,016$ & $0,609 * *$ & 1 & & & & & \\
\hline Cigarros/vida (n) & $-0,015$ & $-0,001$ & $0,704^{* *}$ & $0,728^{* *}$ & 1 & & & & \\
\hline $\begin{array}{l}\text { Dentes } \\
\text { presentes ( } \mathrm{n})\end{array}$ & 0,031 & $-0,001$ & $-0,013$ & $-0,108^{* *}$ & $-0,039$ & 1 & & & \\
\hline IP (freqüência) & 0,006 & $-0,083$ & 0,000 & $-0,055$ & $-0,049$ & $0,606^{* *}$ & 1 & & \\
\hline SS (freqüência) & $-0,022$ & $-0,030$ & 0,049 & $-0,012$ & $0,225^{* *}$ & $0,225^{* *}$ & $0,140^{* *}$ & 1 & \\
\hline PS (média) & $-0,010$ & $-0,000$ & 0,038 & $0,115^{* *}$ & 0,067 & $-0,228^{* *}$ & $-0,123^{* *}$ & $0,306^{* *}$ & 1 \\
\hline
\end{tabular}

${ }^{* *} \mathrm{p}<0,01$ - correlação de Pearson; PS - profundidade de sondagem; IP - índice de placa; SS - sangramento a sondagem; $\mathrm{n}$ - número / $p<0.01$ - Pearson's correlation;PPD - periodontal pocket depth;IP - plaque index; SS - bleeding on probing; $n$ - number.
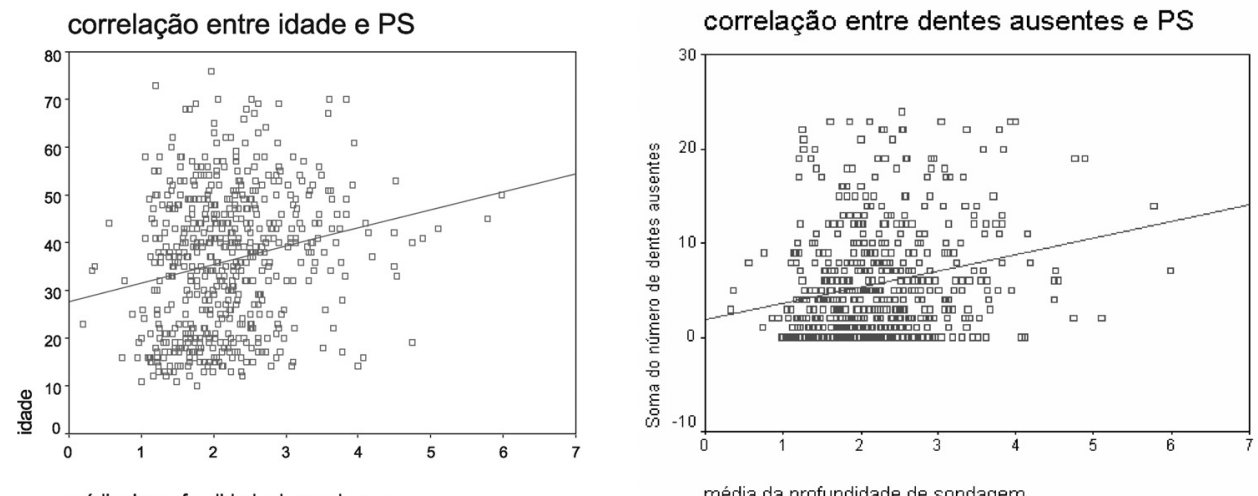

Figura 1 - Gráficos de dispersão entre a variável de interesse profundidade de sondagem (PS) e idade, e entre PS e ausência dentária. Guarulhos, SP, 1999-2003. Teste de correlação de Pearson. Figure 1 - Dispersal graphics between periodontal pocket depth (PPD) and age, and between PPD and dental loss. Guarulhos, SP, 1999-2003. Pearson's correlation test

Tabela 4 - Correlação entre profundidade de sondagem (PS) e dados da anamnese. Guarulhos, SP, 1999-2003. Table 4 - Correlation between periodontal probing depth and clinical information. Guarulhos, SP, 1999-2003.

\begin{tabular}{|c|c|c|c|c|c|c|}
\hline & $\begin{array}{l}\text { Média } \\
\text { de PS }\end{array}$ & $\begin{array}{l}\text { Uso de anti- } \\
\text { séptico }\end{array}$ & $\begin{array}{l}\text { Irritação } \\
\text { gengival }\end{array}$ & $\begin{array}{c}\text { Sangramento } \\
\text { gengival }\end{array}$ & $\begin{array}{c}\text { Teste } \\
\text { glicêmico }\end{array}$ & Tabaco \\
\hline PS (média) & 1 & & & & & \\
\hline Uso de anti-séptico & $0,112^{* *}$ & 1 & & & & \\
\hline Irritação gengival & 0,084 & 0,60 & 1 & & & \\
\hline Sangramento gengival & $0,170^{* *}$ & 0,048 & $0,327^{* *}$ & 1 & & \\
\hline Teste glicêmico & $0,154^{* *}$ & 0,069 & $-0,049$ & 0,036 & 1 & \\
\hline Tabaco & $0,118^{* *}$ & 0,015 & 0,043 & $-0,086$ & $0,112^{* *}$ & 1 \\
\hline
\end{tabular}

Teste de correlação de Pearson * $\mathrm{p}<0,05 ;{ }^{* *} \mathrm{p}<0,01$

Pearson's correlation test ${ }^{*} p<0.05 ;{ }^{* *} p<0.01$ 
Tabela 5 - Associações entre o valor referência de profundidade de sondagem (PS $>3 \mathrm{~mm}$ ), sangramento a sondagem e dados da anamnese. Guarulhos, SP, 1999-2003. Análise de regressão multivariada.

Table 5 - Association between periodontal probing depth cut-off (PPD>3mm), bleeding on probing and clinical information. Guarulhos, SP, 1999-2003. Multivariate regression analysis.

\begin{tabular}{lcc}
\hline Variáveis & \multicolumn{2}{c}{ Profundidade de Sondagem } \\
\hline & Beta & Significância \\
Idade & 0,201 & $\mathrm{p}<0,01$ \\
Sangramento a sondagem & 0,169 & $\mathrm{p}<0,01$ \\
Hábito de fumar & 0,087 & $\mathrm{p}<0,05$ \\
Teste glicêmico & 0,114 & $\mathrm{p}<0,01$ \\
Uso de anti-séptico & 0,087 & $\mathrm{p}>0,05$ \\
Irritação gengival & 0,073 & $\mathrm{p}>0,05$ \\
\hline
\end{tabular}

brasileira têm sido avaliados periodicamente por levantamentos epidemiológicos conduzidos por órgãos governamentais em amostras municipais representativas. Entretanto, o mesmo não tem ocorrido freqüentemente em relação à saúde bucal e periodontal. Deste modo, associando-se a carência de dados nacionais e a importância do município dentro do Estado de São Paulo, o objetivo do presente estudo foi determinar a condição periodontal de uma amostra de conveniência de moradores de Guarulhos. E, a partir da análise de prontuários clínico-odontológicos, sua possível associação com fatores de risco.

A população estudada foi relativamente jovem (36,1 \pm 14,5 anos de idade), o que está de acordo com os dados do último censo ${ }^{2}$, e predominantemente do sexo feminino $(72,1 \%)$, como observado em outros estudos realizados em universidades $^{8,18,19}$. Com poucas exceções como o estudo de Segundo et al. ${ }^{20}$, a literatura evidencia maior prevalência de doença periodontal em afrodescendentes ${ }^{11}$. Semelhante aos dados do $\mathrm{IBGE}^{2}$, que também se baseiam no sistema de autoclassificação, no presente estudo foi encontrada uma população predominantemente branca $(71,3 \%)$, fato que pode ter contribuído para a baixa prevalência de doença periodontal observada.

A condição socioeconômica também tem sido associada a doença periodontal $^{13,15}$. Nos países em desenvolvimen- to, foi relatada presença de grande quantidade de biofilme e cálculos dentários, mesmo em crianças ${ }^{10,21,22}$. No presente estudo, a maioria da população, $37,7 \%$, originou-se da zona leste, região de exclusão socioeconômica de Guarulhos. E, nesta região, foi observada a maior prevalência de doença periodontal, com base no valor referência PS > 3mm. Paralelamente, a redução na renda familiar também parece comprometer os hábitos de higiene bu$\mathrm{cal}^{12}$. No presente estudo, a freqüência média de escovações foi de $2,7 \pm 0,9$ vezes/ dia. Estes achados estão de acordo com Abbeg $^{13}$, que observou uma média de escovação de 3 vezes ao dia em uma amostra de conveniência composta por adultos da Grande Porto Alegre.

Diversos estudos apontaram maior comprometimento periodontal em crianças $^{21}$, adultos ${ }^{8,23}$ e idosos ${ }^{24,25}$ do sexo masculino. Todavia, no presente estudo a análise entre os sexos não demonstrou diferenças com significância estatística em relação à condição periodontal (Tabela 1).

$\mathrm{Na}$ literatura não existe um consenso internacional sobre os critérios que definem doença periodontal. Esta ausência de padronização limita a comparação de resultados e justifica parcialmente a discrepância de dados entre diferentes populações (Quadro 1). A AAP ${ }^{12}$ baseou a ocorrência de doença na presença de PS $>3 \mathrm{~mm}$, em pelo menos um sítio periodontal. Provavelmente, a adoção no presente estudo de critéri- 
os sítio-específicos seria acompanhada de aumento na prevalência de doença periodontal, pois mesmo na presença de número elevado de bolsas periodontais, os valores de PS tendem a se tornar diluídos, quando da obtenção de médias.

No presente estudo, a média populacional de PS foi $2,2 \pm 0,8 \mathrm{~mm}$ e a prevalência de doença periodontal de $13,5 \%$. Silvério ${ }^{19}$ avaliou 326 indivíduos da região do Vale do Paraíba e observou valores médios de PS que variaram de $2,47 \mathrm{~mm}$ a $3,29 \mathrm{~mm}$ de acordo com as faces dentárias. Já Craig et al. ${ }^{26}$ encontraram média de PS de 2,6mm para o grupo de 58 hispânicos. No estudo de Machion et al. ${ }^{8}$ a maior prevalência observada, $57,3 \%$, foi de bolsas periodontais de $3 \mathrm{~mm}$ de profundidade. PS $\geq 4 \mathrm{~mm}$ foi encontrada em 14,3\% dos trabalhadores de 18 a 64 anos nos Estados Unidos da América ${ }^{9}$. Em Hong Kong, Lo et al. ${ }^{27}$ observaram em idosos, prevalência de bolsas rasas de $37 \%$; enquanto na Colômbia Orozco et al. ${ }^{16}$ (2004) encontraram $11,2 \%$ de bolsas periodontais com no mínimo $5,5 \mathrm{~mm}$ de profundidade analisando diferentes faixas etárias. Baelum et al. ${ }^{14}$ encontraram menos de $10 \%$ dos sítios dentais com PS $\geq 4$ $\mathrm{mm}$. Finalmente, no projeto SB Brasil $2003^{7}$, na região sudeste, $10,13 \%$ da população entre 35 e 44 anos de idade apresentaram bolsas periodontais de $4-5 \mathrm{~mm}$; todavia, os autores já ressaltaram a influência da perda dentária sobre a prevalência de doença periodontal a partir dos 35 anos de idade. Apesar dos diferentes tipos de estudo e dos critérios de definição de doença (Quadro 1), em termos gerais os dados prevalentes de doença periodontal da população de Guarulhos foram inferiores.

Além da PS, outros parâmetros são utilizados para caracterizar a condição periodontal, como hábitos de higiene bucal, presença de biofilme supragengival, inflamação gengival e sangramento à sondagem $^{4,28}$. No presente estudo, as freqüências médias de IP e SS foram 75,3\% e 34,1\%, respectivamente. Em um estudo de adultos realizado na Tanzânia ${ }^{14}, 90 \%$ das super- fícies dentais apresentaram biofilme e 30$40 \%$ apresentaram SS. Níveis similares de inflamação (50\%) foram observados por Funosas et al. ${ }^{29}$ na Argentina. E, no projeto SB Brasil $2003^{7}$ na região sudeste $9,74 \%$ da população entre 35 e 44 anos de idade apresentaram sangramento gengival. No presente estudo, foi observada correlação entre IP e SS $(r=0,140$ e $\mathrm{p}<0,01)$. Estes resultados estão de acordo com outros es$\operatorname{tudos}^{21,30}$, que encontraram associação entre presença de biofilme dental e inflamação gengival. Observou-se ainda correlação positiva entre SS e PS ( $\mathrm{r}=0,306$ e $\mathrm{p}<$ 0,01 ) fato previamente demonstrado por outros estudos ${ }^{20,31}$.

O presente estudo ainda mostrou uma correlação fraca $(r=0,223, p<0,01)$ entre PS $>3 \mathrm{~mm}$ e dentes ausentes. Brennan et al. ${ }^{32}$ concluíram que indivíduos que possuíam de 1 a 21 dentes perdidos tinham 1,29 vez mais bolsas periodontais do que aqueles com dentição completa. Foi observada correlação positiva entre PS e idade $(\mathrm{r}=0,198$ e $\mathrm{p}<0,01)$, associação esta já relatada pelos estudos de Machion et al.,19. Adicionalmente, no presente estudo, o fator idade também esteve correlacionado com o número de dentes ausentes ( $\mathrm{r}=$ 0,501 e p $<0,01$ ), fato também observado por Baelum et al. ${ }^{14}$ (1986) e Araújo ${ }^{18}$.

Existem evidências que demonstram o efeito negativo do consumo de cigarros (Teng et al. 2003) e do diabetes mellitus ${ }^{10}$ sobre a condição periodontal. Segundo Papapanou $^{17}$, a taxa de risco para doença periodontal avançada foi 2,82 vezes maior para fumantes quando comparados a não fumantes. A prevalência aqui encontrada de indivíduos fumantes foi baixa (13,3\%). Mas quanto maior o tempo de exposição ao hábito de fumar menor a quantidade de dentes presentes $(r=-0,108$ e $\mathrm{p}<0,01)$ e maiores os valores de PS ( $\mathrm{r}=0,115$ e $\mathrm{p}<$ $0,01)$. Todavia, a correlação entre PS e quantidade de cigarros consumidos durante a vida foi apenas marginalmente significativa ( $p=0,065$ ). Neste estudo foram encontrados 2,9\% de diabéticos. Além da baixa prevalência não foi encontrada as- 
sociação entre PS e diabetes mellitus, o que está de acordo com outros estudos conduzidos em populações brasileiras ${ }^{19,33}$.

A análise de regressão apontou para dois fatores de risco previamente estabelecidos na literatura em outras populações, i.e., idade e hábito de fumar. Desta forma, pode-se sugerir a condução de estudos longitudinais, na população de Guarulhos, a fim de se confirmar o papel destes fatores como fatores de risco para a condição periodontal.

Pela carência de dados sobre a saúde bucal da população de Guarulhos, os achados aqui descritos podem contribuir para o esclarecimento sobre a condição periodontal de indivíduos que procuram atendimento na única Universidade da cidade.
Levantamentos desta natureza possibilitarão no futuro um delineamento mais apropriado de estudos longitudinais, imprescindíveis para a caracterização populacional e instauração de métodos preventivos e terapêuticos específicos.

\section{Conclusões}

A população estudada exibiu elevado grau de inflamação gengival e baixo percentual de bolsas periodontais, estando a condição periodontal associada à idade e ao hábito de fumar.

A população estudada foi composta principalmente por mulheres e indivíduos residentes na zona urbana de Guarulhos, oriundos, sobretudo, da zona leste.

\section{Referências}

1. Kumar PS, Griffen AL, Barton JA, Paster BJ, Moeschberger ML. New bacterial species associated with chronic periodontitis. J Dent Res. 2003; 82: 338-44.

2. Instituto Brasileiro de Geografia e Estatística - IBGE. Indicadores Sociais Municipais: uma análise dos resultados da amostra do Censo Demográfico 2000 Brasil e Grandes Regiões. Rio de Janeiro; 2004.

3. Medronho RA. Epidemiologia. Rio de Janeiro: Editora Ateneu; 2003.

4. Araujo MWB, Cortelli SC. Metodologia de pesquisa aplicada à periodontia médica. In: Maria Christina Brunnetti (Org). Periodontia médica: uma abordagem integrada. $1^{\text {a }}$ ed. São Paulo; 2004. p. 89-112.

5. Gjermo, P., Rosing, C. K., Susin, C. \& Oppermann, R. (2002). Periodontal diseases in Central and South America. Periodontol 2000; 29: 70-8.

6. Ministério da Saúde. Secretaria Nacional de Programas Especiais de Saúde. Divisão Nacional de Saúde Bucal. Levantamento epidemiológico em saúde bucal: Brasil, zona urbana, 1986. Brasília: Centro de Documentação do Ministério da Saúde; 1986.

7. Ministério da Saúde. Projeto SB Brasil 2003: condições de saúde bucal da população brasileira 2002 - 2003: resultados principais/ Ministério da Saúde, Secretaria de Atenção a saúde, Departamento de Atenção Básica. Brasília: Ministério da Saúde; 2004.

8. Machion L, Freitas PM, Cesar Neto JB, Nogueira Filho GR, Nociti Jr FH. A influência do sexo e da idade na prevalência de bolsas periodontais. Pesq Odont Bras 2000; 14(1):33-7.
9. Albandar JM. Periodontal diseases in North América. Periodontol 2000 2001; 29: 31-69.

10. Ragghianti MS, Greghi SLA, Lauris JRP, SantAna PAC, Passanezi E. Influência da idade, sexo, placa bacteriana e fumo nas condições periodontais em uma população de Bauru, Brasil. JAppl O Sci 2004; 12(4): 1-12.

11. Borrell LN,Taylor GW, Borgnakke WS, Nyquist LV, Woolfolk MW, Allen DJ, Lang WP.Factors influencing the effect of race on established periodontitis prevalence. $J$ Public Health Dent 2003; 63(1): 20-9.

12. American Academy of Periodontology. Epidemiology of periodontal diseases. J Periodontol 1996 (Annals); 67: 93545.

13. Abbeg C. Oral hygiene habits among Brazilian adults in an urban area of Southern Brazil. Rev Saúde Pública 1997; 31: 586-93.

14. Baelun V, Fejerskov O, Karring T. Oral Hygiene, gingivitis and periodontal breakdown in adult Tanzanians. $J$ Periodontol Res 1986; 21(3): 221-32

15. Gesser HC, Peres MA, Marcenes W. Condições gengivais e periodontais associadas a fatores socioeconômicos. Rev Saúde Pública 2001; 35(3): 289-93.

16. Orozco AH, Franco AM, Ramirez YGO. Periodontal treatment needs in a native island community in Colombia determined with CPITN. Int Dent J2004, 54(2): 73-6.

17. Papapanou PN. Periodontal disease: Epidemiology. $J$ Periodontol (Annals) 1997; 14: 36. 
18. Araújo MVA. Estudo das condições de saúde bucal e necessidades de tratamento em pacientes do curso de odontologia da Universidade Federal do Pará (dissertação de mestrado). Universidade de São Paulo; 2003.

19. Silvério LO. Avaliação das condições periodontais e suas associações a fatores de risco e indicadores de risco em indivíduos da região do Vale do Paraíba - SP (dissertação de mestrado). Unitau; 2004.

20. Segundo TK, Ferreira EF, Costa JE. A doença periodontal na comunidade negra dos Arturo's, Contagem, Minas Gerais, Brasil. Cad Saúde Pública 2004; 20 (2): 596-603.

21. Moraes ES \& Valença AMG. Prevalência de gengivite e periodontite em crianças de 3 a 5 anos na cidade de Aracaju (SE). Ciênc Odontol Bras 2003, 6(4): 87-94.

22. Brown LJ, Johns BA, Wall TP. The economics of periodontal diseases. Periodontol 2000 2002; 29: 223-34.

23. Marques MD, Pinto AT, Pereira AC, Eriksen HM. Prevalence and determinants of periodontal disease in Portuguese adults: results from a multifactorial approach. Acta Odontol Scand 2000, 58(5): 201-6.

24. Pereira AC, Castellanos RA, Silva SRC, Watanabe MGC, Queluz DP, Meneghim MC. Oral health and periodontal status in Brazilian elderly. Bras Dent J 1996, 7(2): 97-102.

25. Mack F, Mojon P, Budtz JE, Kocher T, Splieth C, Schwahn C, Bernhardt O, Gesch D, Kordass B, John U, Biffar R. Caries and periodontal disease of the elderly in Pomerania. Gerodontol 2004; 21(1): 27-36.

26. Craig RG, Boylan R, Yip J, Bamghoye P, Koutsoukos J, Mijares D, Ferrer J, Imam M, Socransky SS, Haffajee AD. Prevalence and risk indicators for destructive periodontal diseases in 3 urban American minority populations. JClin Periodontol 2001; 28: 524-535.
27. Lo EC, Luo Y, Dyson JE. Oral health of institutionalized elderly in Hong Kong. Community Dent Health 2004, 21(3): 224-26.

28. Albandar JM. Global risk factors and risk indicators for periodontal diseases. Periodontol 2000 2002; 29: 177-206.

29. Funosas E, Martinez A, Maestri L, Siragusa M. A case of prepubertal periodontitis and prevalence of gingivitis in a population attending a university clinic in Rosário, Argentina. Acta odontol Latinoam 1999; 12(2): 89-96.

30. Cunha ACP, Chambrone LA. Prevalência de gengivite em crianças de um nível social baixo. Rev Periodontia 1998; 7:6-10.

31. Claffey N, Nylund K, Kiger R, Garrett S, Egelberg J. Diagnostic predictability of scores of plaque, bleeding, suppuration and pocket depths for probing attachment loss: 37 years of observation following initial therapy. $J$ Clin Periodontol 1990; 17: 108-14.

32. Brennan DS, Spencer AJ, Slade GD. Prevalence of periodontal conditions among public-funded dental patients in Australia. Aust Dent J2001;, 46(2): 114-21.

33. Tomita NE, Chinellato LEM, Pernambuco RA, Lauris JRP, Franco LJ, et al. Condições periodontais e diabetes mellitus na população nipo-brasileira. Rev Saúde Pública 2002; 36(5): 1-11.

recebido em: 17/11/05 versão final reapresentada em: 01/03/06 aprovado em: 14/03/06 